\title{
New endovascular techniques for treatment of life-threatening Takayasu arteritis
}

\author{
Marek Kazibudzki ${ }^{1}$, Łukasz Tekieli ${ }^{1}$, Mariusz Trystuła ${ }^{1}$, Piotr Paluszek ${ }^{1}$, Zbigniew Moczulski², Piotr Pieniążek ${ }^{1}$ \\ ${ }^{1}$ Department of Vascular Surgery and Endovascular Interventions, John Paul II Hospital, Krakow, Poland \\ ${ }^{2}$ Centre for Diagnosis, Prevention and Telemedicine, John Paul II Hospital, Krakow, Poland
}

Adv Interv Cardiol 2016; 12, 2 (44): 171-174

DOI: 10.5114 /aic.2016.59369

\section{Introduction}

Takayasu arteritis (TA) is a medium-size and large artery vasculitis of unknown aetiology affecting mainly the aorta and its major branches, usually in young female patients. Histopathology reveals adventitial thickening, focal lymphocytic infiltration of the tunica media and intimal hyperplasia leading to artery stenosis/occlusion. Less commonly media degeneration presents as aneurysmal dilatation [1]. Clinical symptoms arise from systemic inflammation and local vascular complications. Neurological manifestation of the disease includes headache, dizziness, visual disturbance, transient ischemic attack (TIA) and stroke [2]. Takayasu arteritis may be associated with premature mortality among young patients. Mortality is significant (3-11\%) and varies according to geographical location and management strategy. The most frequently reported causes of death include stroke, myocardial infarction, congestive cardiac failure, and peri- and postoperative complications. The majority of patients (23\%) are unable to work, and about $60 \%$ are limited in everyday activities [3, 4].

\section{Case report}

We report the case of a 33-year-old woman who was previously diagnosed on the rheumatology ward in January 2014 due to unexplained weight loss, anaemia and highly elevated systemic inflammation markers (C-reactive protein of $129 \mathrm{ng} / \mathrm{ml}$, erythrocyte sedimentation rate of $107 \mathrm{~mm} / \mathrm{h}$ ). These findings were accompanied by severe dizziness and an extremely low value of systemic blood pressure measured on both arms. Doppler ultrasound (DUS) examination followed by computed tomography angiography (angio-CT; Mar 2014) revealed thickening of the descending aorta wall, near-to-occlusion stenosis of both common carotid arteries (CCA), severe stenosis of the right subclavian artery (RSA) and occlusion of the left subclavian artery (LSA) as well as the right axillary artery. According to the criteria of large vessel vasculitis a diagnosis of TA was made [2] and the patient was initiated on a combination therapy of cyclophosphamide, prednisolone and hydrocortisone. Within the next 6 months, she was hospitalized several times due to persistent neurological symptoms including dizziness and drop attacks. In December 2014 clinical deterioration with increased neurological symptoms occurred. The patient was unable to adopt an upright position because of severe dizziness; moreover, right-hemisphere TIA occurred. She was admitted to our Vascular Surgery Department in January 2015. After complex non-invasive diagnostic evaluation and multidisciplinary team (neurologist, vascular surgeon, cardiologist, angiologist) consultation she was scheduled for invasive aortic arch angiography in terms of endovascular treatment. Via right femoral access, a $6 \mathrm{Fr}$ diagnostics pigtail catheter was introduced. Angiography revealed: occlusion of the LSA, occlusion of the left CCA, significant narrowing of the innominate artery (IA), long, 95\% stenosis of right CCA, and $80 \%$ stenosis of the RSA (Figure $1 \mathrm{~A}$ ). Cerebral filling was extremely poor, mainly from collateral circulation (Figure 1 B). The right vertebral artery was the only patent vessel supplying the brain. In accordance with ischemic symptoms and predicted procedure feasibility it was decided to perform angioplasty of vessels supplying the right hemisphere. On diagnostics, an Imager II Bern 5 Fr catheter, V-18 Control Wire (0.0018 in) was navigated through the IA to the RSA. The diagnostic catheter was then replaced with a $40^{\circ}$ angled $8 \mathrm{Fr}$ Mach1 Peripheral Guide Catheter (all above from Boston Scientific, Natick, MA, USA). The right CCA and ICA were wired with a coronary BMW 0.014 in wire (Abbott Vascular, Santa Clara,

\section{Corresponding author:}

Łukasz Tekieli MD, Department of Vascular Surgery and Endovascular Interventions, John Paul II Hospital, 80 Prądnicka St, $31-202$ Krakow, Poland, phone: +48 607414 446, e-mail: Itekieli@szpitaljp2.krakow.pl

Received: 3.12.2015, accepted: 21.12.2015. 
CA, USA) and predilatation of the right CCA with a $2.5 \times$ $20 \mathrm{~mm}$ coronary balloon catheter was performed (two inflations at $12 \mathrm{~atm}$ and $14 \mathrm{~atm}$ for $20 \mathrm{~s}$ each). FilterWire EZ (Boston Scientific, Natick, MA, USA), a distal neuroprotection device, was introduced and opened in the right ICA. Two inflations at $6 \mathrm{~atm} / 60 \mathrm{~s}$ using a $5.0 \times 60 \mathrm{~mm}$ IN PACT Admiral Drug Eluting Balloon (DEB; Medtronic, Minneapolis, MN, USA) were performed in the right CCA covering the whole stenotic segment (Figure $1 \mathrm{C}$ ). In spite of long inflations the patient complained only of temporary inflation-related right-side neck pain. As significant dissection of the proximal and middle part of the right CCA (RCCA) occurred (Figure $1 \mathrm{D}$ ), two self-expandable stents (Precise PRO $8.0 \times 40 \mathrm{~mm}$ distally, $9.0 \times 30 \mathrm{~mm}$ proximally; Cordis Fremont, CA, USA) were implanted, covering the dissected segment as well as the artery ostium and bifurcation with the RSA. Postdilatation with a $5.0 \times 20 \mathrm{~mm}$ balloon catheter $(2 \times 12 \mathrm{~atm} / 20 \mathrm{~s})$ was done (Figures 1 E, F). The next target was the RSA. Stent struts covering the RSA ostium were crossed with a 0.014 in HT BMW 0.014 in wire and then predilated with a $2.0 \times 20 \mathrm{~mm}$ balloon catheter. A coronary, drug-eluting stent (DES, Xience Pro $4.0 \times 23 \mathrm{~mm}$ (Abbott Vascular, Santa Clara, (A, USA)) was implanted at $18 \mathrm{~atm} / 30 \mathrm{~s}$ and post-dilated with a $5.0 \times 20 \mathrm{~mm}$ balloon catheter at $14 \mathrm{~atm} / 40 \mathrm{~s}$ (Figure $1 \mathrm{G}$ ). A filter from the RICA was retrieved very easily. In control angiography correct location of stents with no residual stenosis and significant blood flow improvement to both hemispheres were recorded (Figures $1 \mathrm{H}, \mathrm{I}$ ). Within the next $12 \mathrm{~h}$ significant improvement of general status and reduction of neurological symptoms were observed. The patient was discharged after 4 days of hospitalization on dual antiplatelet therapy (aspirin $75 \mathrm{mg}$ /daily permanently and clopidogrel $75 \mathrm{mg} /$ daily for 6 months). Control DUS performed 4 weeks later demonstrated good flow through implanted stents with no signs of restenosis. Three and 8 months after the procedure, control angio-CT revealed patency of all stents and
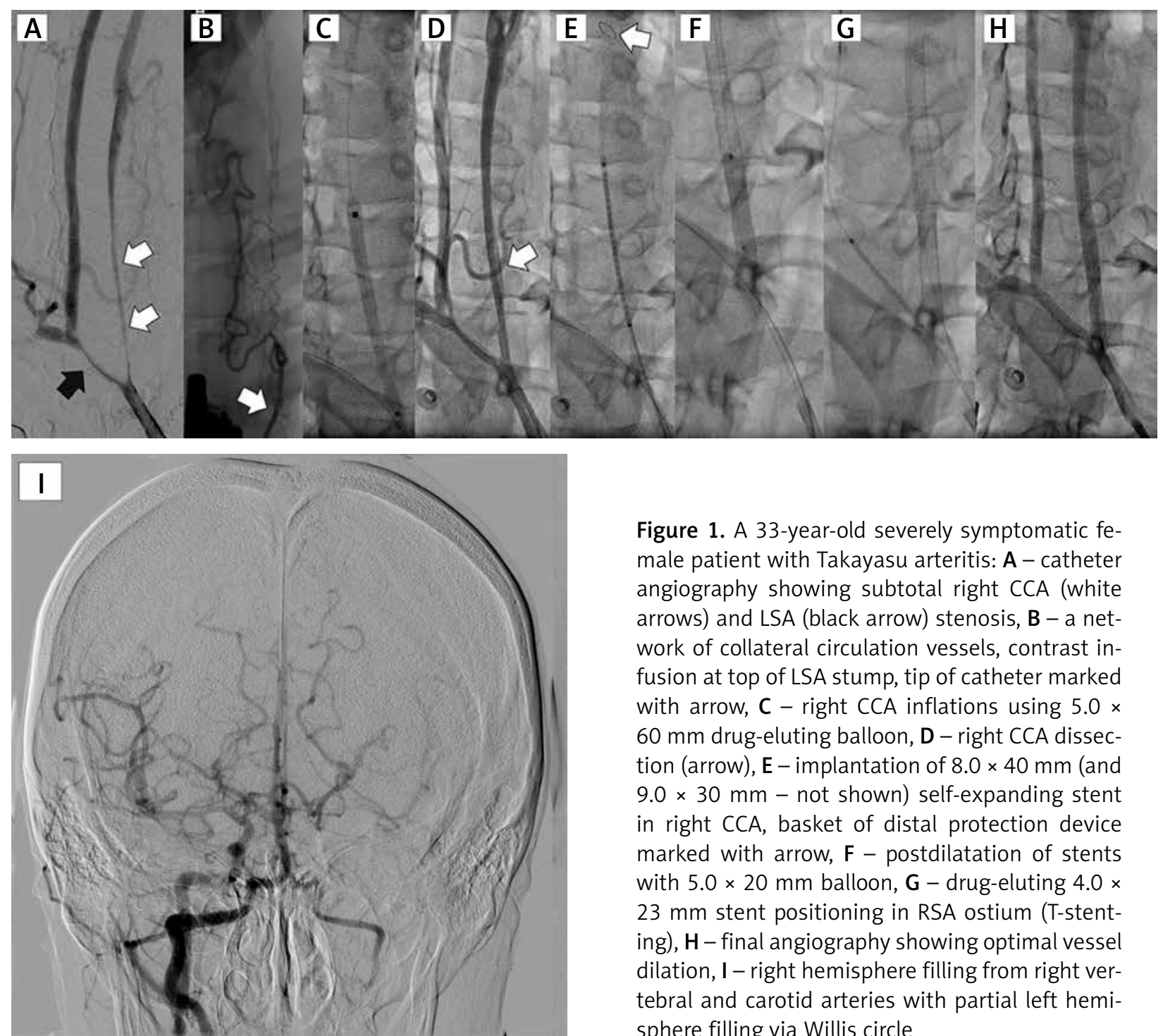

Figure 1. A 33-year-old severely symptomatic female patient with Takayasu arteritis: A - catheter angiography showing subtotal right CCA (white arrows) and LSA (black arrow) stenosis, B - a network of collateral circulation vessels, contrast infusion at top of LSA stump, tip of catheter marked with arrow, C - right CCA inflations using $5.0 \times$ $60 \mathrm{~mm}$ drug-eluting balloon, $\mathrm{D}$ - right CCA dissection (arrow), $\mathrm{E}$ - implantation of $8.0 \times 40 \mathrm{~mm}$ (and $9.0 \times 30 \mathrm{~mm}-$ not shown) self-expanding stent in right CCA, basket of distal protection device marked with arrow, $\mathbf{F}$ - postdilatation of stents with $5.0 \times 20 \mathrm{~mm}$ balloon, G - drug-eluting $4.0 \times$ $23 \mathrm{~mm}$ stent positioning in RSA ostium (T-stenting), $\mathbf{H}$ - final angiography showing optimal vessel dilation, I - right hemisphere filling from right vertebral and carotid arteries with partial left hemisphere filling via Willis circle 

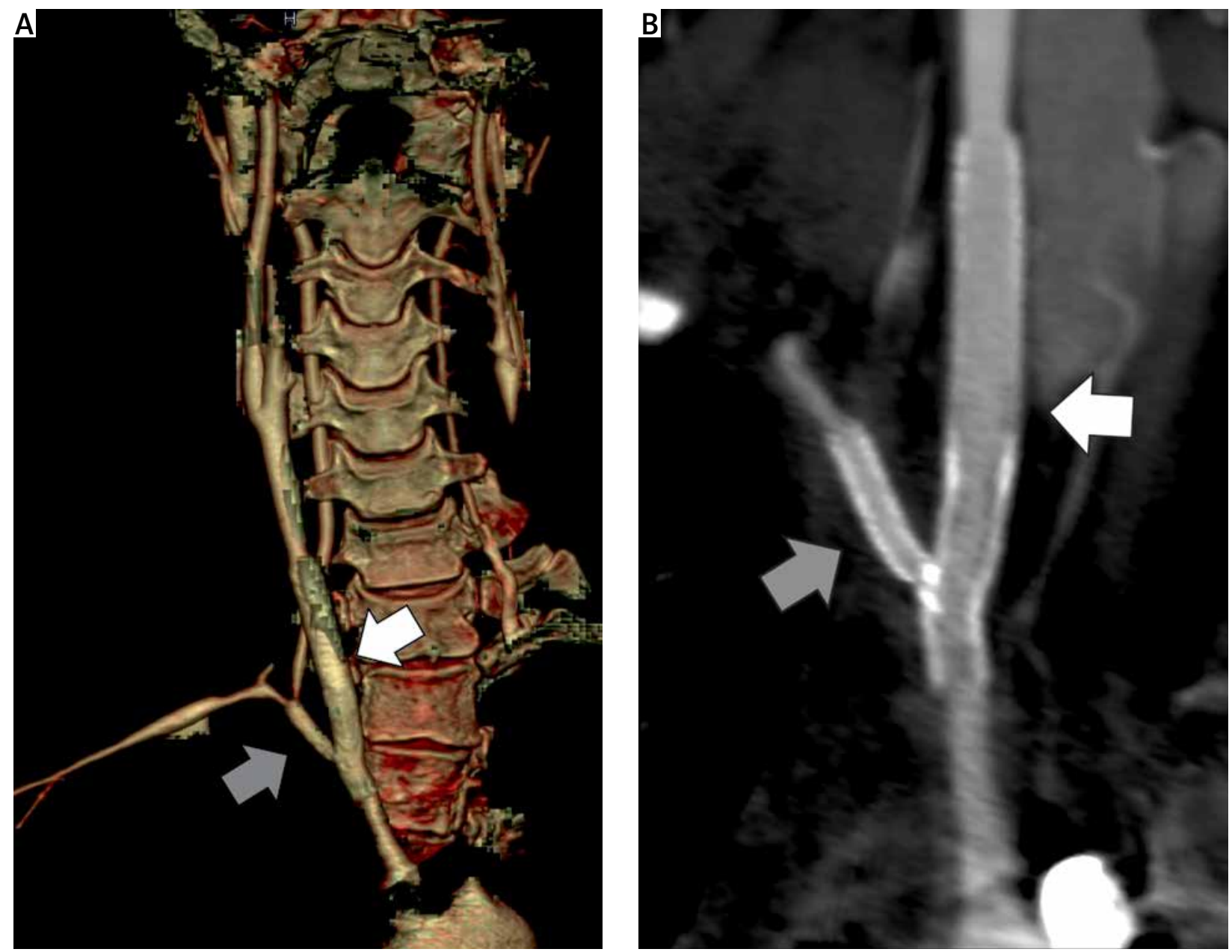

Figure 2. Control angio-CT of the patient performed 7 months after procedure. A - 3D reconstruction showing all stents' patency and no new stenotic lesions of treated vessels. B - 2D cross-section demonstrating undamaged right CCA (white arrow) and RSA (grey arrow) stents' structure

no new stenotic lesions of treated vessels (Figure 2). Up to now, the patient has remained in good clinical status without any neurological disorders.

\section{Discussion}

Surgical bypass arteries reconstruction has been shown to be superior to endovascular treatment in TA [5]. However, this relates usually to lower limb and renal arteries. The higher risk of serious early and postprocedural surgical complications should also be considered $[3,6,7]$. With intensive technological development, endovascular treatment has been introduced as an alternative to surgery for arterial stenotic lesions in most vascular territories. Endovascular treatment of symptomatic TA has been found to be safe and very effective with an acceptable 30day complication rate of $7.1 \%$ as reported by Wrotniak et al. [8]. However, it has been shown that in-stent stenosis remains the main issue in both the surgical and the endovascular approach [7, 9]. Different mechanisms of artery stenosis development in non-atherosclerotic vas- culitis, such as TA, including chronic intramural inflammation, suggest that the use of drug-eluting systems might be an optimal way to deal with severe intimal hyperplasia, leading to early and late restenosis affecting $>30 \%$ of treated vessels $[7,9,10]$. Besides typical in-stent stenosis, external stent compression by progressive vessel wall fibrosis and calcification has been described [10]. In fact, there is insufficient information on the optimal endovascular treatment of TA, especially concerning use of drug-eluting devices in the supra-aortic territory. Our case shows that such therapy may be safe and effective, including in medium-term observation.

We used DEB before stent implantation in the RCCA because there have been no drug-eluting self-expanding stents available on the market so far. This strategy gives a higher probability of restenosis avoidance. On the other hand, the use of drug-eluting stents has been shown to increase the risk of early and late in-stent thrombosis, which in the carotid territory might have devastating sequelae. The experience acquired in coronary interventions supports the strategy of at least 6 months dual 
antiplatelet therapy, as it was implemented in the described case. Coherent systemic treatment with steroids and antiproliferative drugs is crucial in terms of disease inhibition as withdrawal of drugs might be associated with in-stent stenosis [11, 12].

Rigorous follow-up may not be mandatory after carotid angioplasty for atherosclerotic lesion. For the carotid territory, self-expanding stent implantation is associated with $5-7 \%$ risk of significant in-stent stenosis, and it is observed mainly during the first year of follow-up [13]. In TA the risk of restenosis is significantly higher, reaching $>50 \%$ at 5 years, and probably it does not decrease with time [8]. The risk of symptomatic disease progression related to a new stenotic lesion is also very high and exceeds $70 \%$ at 5 years $[8,14]$. These findings support watchful and frequent DUS and angio-CT examination that have been performed in our patient. As DUS is not a perfect image modality for evaluation of proximal segments of aortic arch arteries, it was decided to carry out regular angio-CT scanning. This examination enables optimal visualisation of all supra-aortic arteries including not only the vessel wall but also stent structure in terms of potential stent deformation/fracture.

Outcomes of vascular intervention in TA may be improved by detailed preoperative assessment including measurement of disease activity, and by ensuring optimal immunomodulatory therapy before and after the procedure [12].

\section{Conclusions}

Multivessel, symptomatic TA may be treated successfully by an endovascular approach using drug-eluting balloons and stents with good immediate and mid-term results. Watchful follow-up is essential for early restenosis/disease progression diagnosis.

\section{Conflict of interest}

The authors declare no conflict of interest.

\section{References}

1. Kerr GS, Hallahan CW, Giordano J, et al. Takayasu arteritis. Ann Intern Med 1994; 120: 919-2.

2. Arend WP, Michel BA, Bloch DA, et al. The American College of Rheumatology 1990. Criteria for the classification of Takayasu arteritis. Arteritis Rheum 1990; 33: 1129-34.

3. Perera AH, Mason JC, Wolfe JH. Takayasu arteritis: criteria for surgical intervention should not be ignored. Int J Vasc Med 2013; 2013: 618910.

4. Hedna VS, Patel A, Bidari S, et al. Takayasu's arteritis: is it a reversible disease? Case report and literature review. Surg Neurol Int 2012; 3: 132.

5. Kim YW, Kim DI, Park YJ, et al. Surgical bypass vs endovascular treatment for patients with supra-aortic arterial occlusive disease due to Takayasu arteritis. J Vasc Surg 2012; 55: 693-700.
6. Ham SW, Kumar SR, Rowe VL, et al. Disease progression after initial surgical intervention for Takayasu arteritis. J Vasc Surg 2011; 54: 1345-51.

7. Saadoun D, Lambert M, Mirault T, et al. Retrospective analysis of surgery versus endovascular intervention in Takayasu arteritis: a multicenter experience. Circulation 2012; 125: 813-9.

8. Wrotniak L, Kabłak-Ziembicka A, Przewłocki T, et al. Long-term experience in patients undergoing endovascular revascularization procedures for symptomatic Takayasu arteritis. J Rare Cardiovasc Dis 2013; 1: 8-13.

9. Horie N, Hayashi K, Morikawa M, et al. Restenosis after endovascular PTA/stenting for supra-aortic branches in Takayasu aortitis: report of three cases and review of the literature. Acta Neurochir (Wien) 2011; 153: 1135-9.

10. Spacek M, Zimolova P, Veselka J. Takayasu arteritis: use of drug-eluting stent and balloon to treat recurring carotid restenosis. J Invasive Cardiol 2012; 24: E190-2.

11. Hu J, Huang $H$, Zhang $X$, et al. Stent placement for treatment of long segment ( $\geq 80 \mathrm{~mm}$ ) carotid artery stenosis in patients with Takayasu disease. J Vasc Interv Radiol 2012; 23: 1473-7.

12. Perera AH, Youngstein T, Gibbs RG, et al. Optimizing the outcome of vascular intervention for Takayasu arteritis. Br J Surg 2014; 101: 43-50.

13. Brott TG, Halperin JL, Abbara S, et al. ASA/ACCF/AHA/AANN/ AANS/ACR/ASNR/CNS/SAIP/SCAI/SIR/SNIS/SVM/SVS guideline on the management of patients with extracranial carotid and vertebral artery disease. J Am Coll Cardiol 2011; 57: 1002-44.

14. Maksimowicz-McKinnon K, Clark TM, Hoffman GS. Limitations of therapy and a guarded prognosis in an American cohort of Takayasu arteritis patients. Arteritis Rheum 2007; 56: 1000-9. 